\title{
An Update on Postoperative Opioid Use and Alternative Pain Control Following Spine Surgery
}

\author{
Kevin Berardino, BS ${ }^{1}$, Austin H. Carroll, BA ${ }^{1}$, Alicia Kaneb, BA ${ }^{1}$, Matthew D. Civilette, BS ${ }^{1}$, William F. Sherman, MD, \\ $\mathrm{MBA}^{2}$, Alan D. Kaye, MD, $\mathrm{PhD}^{3}$ \\ ${ }^{1}$ Georgetown University School of Medicine, Washington D.C., ${ }^{2}$ Department of Orthopedic Surgery, Tulane University, New Orleans, LA, 3 \\ Department of Anesthesiology, Louisiana State University Shreveport, LA \\ Keywords: dependence, addiction, postoperative, pain control, opioids, spine surgery \\ https://doi.org/10.52965/001c.24978
}

\section{Orthopedic Reviews}

Vol. 13, Issue 1, 2021

\begin{abstract}
Opioids are commonly prescribed postoperatively for pain control, especially in spine surgery. Not only does this pose concerns for potential abuse, but it also has been shown to worsen certain outcomes. Risk factors for increased use include preoperative opioid use, female sex, psychiatric diagnoses, and drug and alcohol use. Over the past few decades, there have been increasing efforts mostly spearheaded by governmental agencies to decrease postoperative opioid use via opioid prescription limitation laws regulating the number of days and amounts of analgesics prescribed and promotion of the use of enhanced recovery after surgery (ERAS) protocols, multimodal pain regimens, epidural catheters, and ultrasound-guided peripheral nerve blocks. These strategies collectively have been efficacious in decreasing overall opioid use and better controlling patients' postoperative pain while simultaneously improving other outcomes such as postoperative nausea, vomiting, and length of stay. With an aging population undergoing an increasing number of spinal surgeries each year, it is now more important than ever to continue these efforts to improve the quality and safety of pain control methods after spinal surgery and limit the transition of acute management to the development of opioid dependence and addiction long-term.
\end{abstract}

\section{INTRODUCTION}

\section{OPIOID EPIDEMIC AND SEQUELAE}

Opioid misuse has reached epidemic status in the United States. In 2008, more Americans died from opioid overdose than from automobile accidents. ${ }^{1}$ Studies have placed the number of daily deaths from an opioid overdose at over 220 people per day. The crisis has become widely regarded as a public health emergency with over 81,000 deaths, according to the Centers for Disease Control in 2020 alone in the United States from opioid overdoses. These sobering facts have led to Congress passing an $\$ 8$ billion SUPPORT for Patients and Communities Act to address the nation's opioid overdose epidemic. $^{2-4}$

In 2017, opioids made up nearly $70 \%$ of all drug overdoses, and most were from prescriptions. ${ }^{1,5}$ The rate of drug overdose mortality by 2017 was five times that of $1999 .{ }^{6} \mathrm{Un}-$ fortunately, accidental/non-intentional overdose is not uncommon. ${ }^{4}$ Most illegal opioid use starts from legal prescriptions, pointing to the critical responsibility that healthcare workers, in particular physicians and prescribers, may have in this crisis. ${ }^{5}$
Opioid misuse contributes not only to mortality via overdose. Still, it has also been shown to increase the risk of acute coronary syndrome . ${ }^{2}$ Between 2015 and 2020, infective endocarditis related to injection drug use also nearly doubled, most notably among younger patients without significant comorbidities. ${ }^{2}$ Following surgery, chronic opioid use is additionally associated with a greater likelihood of complications such as wound dehiscence and surgical site infection and postoperative constipation that is more resistant to traditional laxative therapies. ${ }^{7}$

\section{OPIOID PRESCRIPTIONS AND SURGERY}

In the United States, 98\% of patients have been prescribed opioid medications following surgery. ${ }^{8}$ While many of these patients do not use opioid medicines before surgery, most are routinely prescribed them postoperatively. ${ }^{8}$ The majority of patients receiving postoperative opioid prescriptions receive an excess of pills, and $40 \%$ of these prescriptions are never properly disposed of, creating an opportunity for diversion. ${ }^{1}$ To illustrate the excess of opioids in circulation, one study found that $88 \%$ of total knee arthroplasty (TKA) patients were prescribed more opioid pills than they used or

\footnotetext{
a Corresponding author: Kevin Berardino, BS Georgetown University School of Medicine, Washington DC, email: kmb364@georgetown.edu
} 
required. ${ }^{9}$

Among surgical specialties, orthopedic and neurosurgery procedures tend to have the highest rates of opioid use, and interventional spinal procedures fall at the intersection of these specialties. ${ }^{10}$ Over the past three decades, the diagnoses and treatment of spinal pathologies, such as lumbar stenosis and spondylolisthesis, have increased as the United States experiences an aging population. ${ }^{11}$ Meanwhile, opioid use following common procedures such as spinal and/or lumbar fusion operations is strikingly high. For the most part, physicians prescribe regimens that vary in dosage and duration without a standard regimen in place. ${ }^{12}$

Orfield et al. compared chronic opioid prescriptions following 50 common orthopedic procedures and found that 8 of the 10 surgeries with the highest rates of long-term opioid prescription usage were spine surgeries, consistent with the invasive nature of spinal surgery. ${ }^{9}$ Opioid overuse in spine surgery has also been demonstrated by a trial which showed an increase in post-surgical opioid dependence from $0 \%$ to $47.8 \%$ of patients who underwent surgical fusion for degenerative scoliosis from 2001-2015. ${ }^{13}$

There are varying degrees of invasiveness among procedures within spine surgery, subsequently impacting opioid prescriptions and usage. For example, the spinal fusion of four or more levels, compared to one to three, is associated with greater opioid use after surgery. ${ }^{5}$ In lumbar fusion, there is increasing evidence showing that minimally invasive surgery (MIS) may lead to earlier opioid independence at 2 vs. 4 weeks and decreased postoperative opioid use. ${ }^{14-16}$ Similarly, Schoenfeld et al. showed that less intensive discectomy and decompression procedures had a greater likelihood of patient opioid discontinuation than lumbar interbody arthrodesis. ${ }^{17}$ Thus, specific surgical characteristics are crucial to keep in mind when considering postoperative opioid use.

The etiology of the opioid endemic surrounding spine surgery may also be influenced by the multidisciplinary nature of the patient care process. Although surgeons accounted for $69 \%$ of opioid prescriptions in the first three months after surgery, they accounted for only $11 \%$ of opioid prescriptions at 9-12 months, whereas primary care physicians accounted for $53 \% .{ }^{18}$ Postoperative gaps in communication between care teams can lead to lack of ownership of opioid management, potentially leading to excess refills or misinformation regarding intended course longevity. ${ }^{19}$

Opioid pharmacology and physiology understanding have evolved in recent decades since identifying the opioid receptor over four decades ago. Opioid prescriptions or any exogenous opioid will shut down endogenous opioid production, resulting in reduced levels of endorphins, enkephalins, and dynorphins. This process, which takes weeks and months, results in opioid dependence. In addition, without exogenous opioid delivery, patients will exhibit central nervous system hyperarousal states or what is commonly known as withdrawal symptoms. Thus, acute pain management can directly affect patients and their resultant development of opioid dependence and addiction. Further, it is the basis of why the government has established policies throughout the United States in all fifty states limiting opioids in the setting of acute pain manage- ment to reduce transition to opioid dependence and addiction states.

\section{IMPACTS ON OUTCOME AND STAY}

The clinical significance of increased opioid utilization has been shown to impact outcomes, especially ongoing opioid use before surgery negatively. Increased 1-year reoperations evidence this, emergency department (ED) visits, epidural and facet joint injections, wound complications, infections, neurological complications, acute renal failure, and venous thromboembolism rates following cervical fusion in patients on chronic opioid therapy (COT) preoperatively. ${ }^{20}$ One animal model showed that opioid use before and after lumbar fusion led to the delayed remodeling of the fusion at 6 weeks - one potential explanation for higher rates of reoperation in patients on COT preoperatively. ${ }^{21,22}$ Patients who receive preoperative COT are also less likely to return to work after spine surgery, affecting their quality of life and further confounding postoperative outcomes. ${ }^{22,23}$ Additionally, multiple studies have shown COT patients to have an increased length of stay. ${ }^{24,25} \mathrm{Kha}$ et al. demonstrated an increase of 1.1 days for every 100 morphine milligram equivalents (MME), which directly impacts healthcare utilization and the outcomes of the individual patients. ${ }^{25}$ Finally, another concerning outcome is the development of chronic opioid use following spinal procedures. Cook et al. found that the development of chronic opioid use following common spinal procedures to be $15-18 \%$ in opioid-naïve individuals and $50-64 \%$ in chronic opioid users. ${ }^{26}$ This illustrates both the magnitude of opioid use following spinal procedures in general and also the risk that preoperative COT poses for long-term use.

\section{RISK FACTORS FOR INCREASED OPIOID USE FOLLOWING SPINE SURGERY}

\section{PRE-OP USAGE AND POSTOPERATIVE USE/DURATION}

Just as preoperative opioid use impacts specific outcomes, it also plays a role in postoperative consumption. However, the term "preoperative opioid use" has varying criteria; some studies define it as self-reported opioid use or prescription availability at the time of surgery, while others include date ranges up to 365 days before surgery. ${ }^{27}$ Given the variability of this term, preoperative opioid use ranges from $10.2 \%$ to $70 \%$ of patients undergoing spine surgery. ${ }^{27,28} \mathrm{Re}-$ gardless, preoperative opioid use has been widely associated with greater postoperative opioid use following spine surgery. ${ }^{20,21,23,25,29-32}$ Across all major spine surgery, Dunn et al. showed that $52 \%$ of patients on COT preoperatively still used opioids 12 months postoperatively compared to $18.3 \%$ of opioid-naïve individuals. ${ }^{33}$ Following one and two-level posterior lumbar fusions, one study found $87.4 \%$ of patients on preoperative COT were still using opioids for at least 12 months. ${ }^{21}$ Following lumbar decompression without fusion, transforaminal or posterior interbody fusion, anterior cervical discectomy and fusion, cervical disc arthroplasty, and posterior lumbar fusion spine procedures, only $63-68 \%$ of opioid naïve patients fill an opioid prescription, contrasting with the $91-95 \%$ of chronic pre- 
operative users. ${ }^{26}$ Additionally, preoperative opioid users have shown to have a significantly higher opioid fill rate than those who did not use opioids before cervical arthrodesis (45.3\% vs. 6.3\%). ${ }^{30}$ Following spinal decompression, patients on preoperative COT, have shown to receive more opioids while also having a significantly longer postoperative opioid course (7 months) compared to opioid naïve individuals (2.6 months). ${ }^{25}$

\section{SEX DIFFERENCES}

A study of opioid use following spinal surgery found that female sex was associated with higher rates of opioid dependence postoperatively, with a relative risk of $1.166 .{ }^{34} \mathrm{An}$ additional, separate study of patients undergoing lumbar decompression and fusion also found female sex to be an independent risk factor for prolonged opioid use following surgery with an odds ratio of $1.14 .{ }^{35}$ Notably, there was no significant sex difference among preoperative opioid use in this study population. 35

Women may be more likely to be prescribed and therefore use opioid analgesics by healthcare providers. ${ }^{36}$ Additionally, opioid overdose deaths are also more frequent among female patients. ${ }^{36}$ Postoperatively, female patients have reported higher pain levels and required higher doses of morphine as soon as their arrival in the post-anesthesia care unit (PACU). ${ }^{35}$ These findings could perhaps be attributed to chronic pain conditions being more typical among female patients, who may also have increased pain sensitivity. 35

\section{PSYCHIATRIC CONSIDERATIONS}

Psychiatric diagnoses have been associated with higher rates of both pre-and postoperative opioid use. ${ }^{37}$ Anxiety and depression are not only associated with worse surgical outcomes, but they also make a patient less likely to discontinue postoperative opioid use. ${ }^{38}$ Among patients who were not taking opioids before surgery, a diagnosis of post-traumatic stress disorder was associated with an increased likelihood of prolonged postoperative opioid use. ${ }^{12}$

Unfortunately, psychiatric comorbidities may be inadvertently treated with increased opioid refills, prolonging and exacerbating the problem. ${ }^{39}$ Self-confidence and "positive mood" were found to be "the most effective pain relievers," according to a 2017 editorial by the Journal of Bone and Joint Surgery. ${ }^{39}$ Thus, patients suffering from psychiatric disorders may already be predisposed to poor outcomes further impacted by postoperative opioid use and dependence. One study found that regardless of psychiatric diagnosis, preoperative administration of duloxetine, an SNRI antidepressant, led to decreased postoperative pain and improved functional outcomes, pointing to the interrelated nature of physical and psychiatric pain. ${ }^{37}$

\section{SMOKING/ALCOHOL/RECREATIONAL DRUG USE}

Tobacco smoking has been shown to increase preoperative opioid use among female patients undergoing spinal surgery; however, this study did not examine used in the postoperative period. ${ }^{40}$
Alcohol and drug abuse are associated with increased odds of chronic opioid use following any surgery, with odds ratios 1.83 and 3.15 , respectively. ${ }^{41}$ A separate study of patients undergoing spinal surgery concluded that tobacco, alcohol, and drug abuse were risk factors for opioid dependence but did not quantify how great the impact was. ${ }^{33}$

\section{OTHER}

Higher rates of postoperative opioid use are also associated with younger patients and obesity. ${ }^{34,35}$ Furthermore, the likelihood of postoperative opioid use increases with higher dosages, measured in MME. ${ }^{42}$ In other words, prescribing a lower dose of postoperative opioids may help decrease overall postoperative opioid use. ${ }^{42}$ Finally, patients with Health Maintenance Organization insurance plans are less likely to receive opioid prescriptions from an in-office provider. ${ }^{11}$

\section{INTERVENTIONS TO REDUCE USAGE AND THEIR OUTCOMES}

\section{ENHANCED RECOVERY AFTER SURGERY PROTOCOL}

The enhanced recovery after surgery (ERAS) protocol, designed in 1997, is a multimodal approach to surgical care designed to address preoperative, intraoperative, and postoperative stressors to reduce complications, morbidity, and patient pain. ${ }^{43}$ In this regard, most ERAS protocols are designed to have decreased opioid consumption postoperatively, and reduced hospital stays. Since its development, ERAS protocols have been utilized in various surgical specialties, including breast surgery, colorectal surgery, and hepatic surgery. ${ }^{4-46}$ There are designed to be executed by a multidisciplinary team ranging from anesthesiologists, surgeons, nutritionists, occupational therapists, nurses, case managers, and others and is individually tailored to the surgical subspecialty. ${ }^{47}$ By design, ERAS protocols that are accepted in practice have not only been shown to reduce the length of stay (LOS) and opioid consumption postoperatively but as well, demonstrate a reduction in complications, reduction in postoperative costs, and lower patient dissatisfaction rates across a wide variety of surgical subspecialties. ${ }^{44,48-50}$

In recent years, the utility of ERAS with regard to spinal surgery has explicitly come into question as spinal surgeries are particularly invasive and continue to increase in the United States each year. ${ }^{51,52}$ Patients undergoing these surgeries often experience significant postoperative pain, long recovery times, and high rates of complications. ${ }^{53,54}$ ERAS is thus a potential solution to reduce LOS, improve patient outcomes, and reduce postoperative opioid use.

Regarding lumbar surgery specifically, the ERAS society, a group designed to provide expert consensus on ERAS protocol recommendations for specific surgical subspecialties, recently investigated available data and made numerous recommendations. ${ }^{54}$ In the preoperative period, recommendations are focused on preoperative counseling and education, nutrition assessment, cessation of smoking and alcohol, preoperative fasting to include clear fluids up to 2 hours and solid foods up to 6 hours before surgery, and management of preoperative anemia. ${ }^{55}$ Further, the use of 
sedatives for anxiolysis preoperatively is not recommended. In contrast to this, NSAIDs, neuropathic pain medicines, and acetaminophen have been shown in numerous randomized control trials and meta-analyses to reduce opioid use. ${ }^{54,56,57}$

Intraoperatively, the ERAS protocol focuses on antimicrobial skin preparation, intraoperative anesthetics with neuromuscular blockade, and neuraxial techniques such as epidural anesthetics and inhalational agents, the prevention of hypothermia, the use of local and regional anesthetics, and careful fluid and urinary volume management. ${ }^{54}$ Specifically, randomized control trials have shown that intrathecal morphine and epidural anesthetics such as bupivacaine directly reduce opioid use after spine surgery. ${ }^{58-60}$ Direct wound installations with local anesthetics such as ropivacaine and bupivacaine have also been demonstrated to decrease opioid use. ${ }^{61}$

Postoperatively, particular attention is given to nausea control, early mobilization, and postoperative management of drains. ${ }^{54}$ In addition, a multimodal approach is taken to postoperative prescription medication management. Among this approach, acetaminophen, NSAIDs, and neuropathic pain medications, including gabapentinoids, remain the cornerstone of treatment. When used in combination, these drugs dramatically reduce pain, improve patient satisfaction, and reduce opioid consumption and opioidrelated complications. ${ }^{62-64}$ Finally, the ERAS protocol involves continual auditing and improvement based on gathered data to further advance outcomes. ${ }^{54}$ While recommendations are limited to lumbar surgery currently, further prospective research is underway to investigate the benefits and limitations of an ERAS protocol for thoracic and cervical spine surgery.

\section{PRESCRIPTION MEDICATIONS AND MULTIMODAL PAIN REGIMENS}

When used in excess, opioids can have significant adverse side effects, including increased rates of constipation, depression, respiratory disorders, drug overdoses, and others. ${ }^{65-67}$ As a result, multimodal prescription pain regimens have been developed to reduce opioid use and subsequent adverse effects. Current multimodal regimens typically include acetaminophen, NSAIDs, gabapentinoids, muscle relaxants, and potentially local analgesics.

Acetaminophen is an analgesic and antipyretic drug that appears to work via inhibition of prostaglandin synthesis by reducing the conversion of $\mathrm{PGG}_{2}$ to $\mathrm{PGH}_{2}$ and, thus, the ultimate generation of arachidonic acid metabolites and has no known anti-inflammatory effect. ${ }^{68}$ NSAIDs, another cornerstone of multimodal therapy, demonstrate anti-inflammatory, antipyretic, and analgesic effects and rely on the inhibition of cyclooxygenase enzymes that result in the development of inflammatory and pain-inducing prostaglandins. ${ }^{69}$ Neuropathic medications such as pregabalin and gabapentin are also typically included and function through interactions with the alpha ${ }_{2}$-delta subunit of voltage-gated calcium channels located in neurons throughout the nervous system. ${ }^{70}$ These medications are amino acid drugs that reduce pain processing by reducing excitatory amino acids and neuropeptides released during pain signaling. ${ }^{70,71}$ Less consistently included are muscle relaxants such as methocarbamol or diazepam and local anesthetics such as transdermal lidocaine patches that function via prolonging the inactivation of sodium channels within the cell membrane of neurons. ${ }^{72}$

While multimodal approaches have previously been used in orthopedic surgery and other surgical fields, recent research has investigated the use of multimodal approaches involving these drugs specifically in postoperative pain for spine surgery. A multimodal regimen consisting of scheduled doses of acetaminophen, NSAIDs, gabapentin, lidocaine patches, and one of two muscle relaxants was compared to a non-standardized provider-dependent regimen in one retrospective cohort after posterior lumbar surgery. ${ }^{73}$ Opioids were only reserved for breakthrough pain. Opioid use was significantly lower in the multimodal group ( $\mathrm{p}<0.02)$, while pain scores were also significantly lower ( $p<0.001)$. Another series compared IV morphine to a similar multimodal approach and again found a reduction in opioid usage after lumbar surgery. ${ }^{74}$ Mathieson et al. also found a multimodal approach to be associated with statistically significant lower rates of nausea, earlier rates of mobilization, shorter lengths of stay, and lower rates of sedation. ${ }^{75}$

Additionally, in 2016, Bohl et al. investigated a multimodal approach to pain management in cervical spine surgery with similar results. Their series of 239 patients again found decreased length of stay $(\mathrm{p}<0.001)$, decreased narcotic use $(\mathrm{p}<0.001)$ and decreased nausea and vomiting $(p<0.001)$. Other series with cervical, thoracic, and lumbar spine surgery patient populations have also validated these findings. ${ }^{76}$ Although research largely supports multimodal approaches to postoperative prescribing. Further research is necessary to continue drug combination optimization.

\section{EPIDURAL CATHETERS AND PERIPHERAL NERVE BLOCKS}

Although often employed as a multimodal pain regimen that also includes oral and enteral medications, several local techniques have been utilized to reduce postoperative pain and opioid use after spinal surgery. One such technique includes continuous epidural analgesia in which a catheter is used to deploy medications such as bupivacaine or fentanyl into the epidural space. ${ }^{77}$ In one series of 613 patients undergoing scoliosis surgery, patients were randomized to either having an epidural catheter placed intraoperatively that could continually deploy medication or receiving patient-controlled anesthesia (PCA) via a pump that deployed opioid medications. ${ }^{78}$ Pain scores were significantly decreased in the epidural group at all measured time periods compared to the PCA group $(p<0.001)$. This was again shown in a series demonstrating that patients with epidural catheters that deployed bupivacaine and fentanyl required less opioid medication after lumbar surgery. ${ }^{79}$

Other spinal regimens include single-dose epidural medications and intrathecal medications. ${ }^{80}$ Single-dose regimens are applied preoperatively by advancing a needle into the epidural space or administered directly by the surgeon to exposed dura once decompression has been performed. ${ }^{81,82}$ Drugs used include bupivacaine, methylpred- 
nisolone, morphine, clonidine, and many others. Bourke et al. demonstrated that dural application of a single dose of $3 \mathrm{mg}$ of morphine decreased postoperative pain scores compared to controls. ${ }^{83}$ However, a series of 150 patients comparing a single dose of epidural bupivacaine or methylprednisolone versus normal saline found no difference in postoperative pain or opioid use. ${ }^{81}$

Intrathecal administration is typically conducted via direct injection of medication into the dural sac once decompression has been completed. 84 One of the more common medications used for this purpose, morphine, has been demonstrated to suppress the respiratory drive and cause hypoxia. 85 Thus, it is essential to use the lowest dose possible and to monitor respiratory rate and oxygen saturation. However, in one series of 150 patients, $0.2 \mathrm{mg}$ of intrathecal morphine was shown to reduce opioid usage $(p<0.03)$ and was not shown to increase the risk of respiratory depression. ${ }^{84}$ Chan et al. also used 15 micrograms of intrathecal fentanyl in a series of patients. It is absorbed more rapidly due to its lipophilic properties with a lower risk of respiratory compromise. ${ }^{86}$ Another series demonstrated that intrathecal neostigmine, an acetylcholinesterase inhibitor, was able to reduce postoperative opioid use. ${ }^{87}$

Aside from neuraxial anesthesia, peripheral nerve blocks have been utilized in combination with other pain regimens to decrease intraoperative and postoperative pain. One novel technique is the erector spinae plane (ESP) block. Developed in 2016, the ESP block is an interfascial block that targets the dorsal and ventral rami of the spinal nerves as they exit the spinal cord with a local anesthetic. ${ }^{88}$ It is performed at the spinal level (s) undergoing surgery and is guided via ultrasound. Elyazed et al. found that only $13.3 \%$ of patients required intraoperative fentanyl with bilateral ESP blocks vs. 90\% in the control group ( $\mathrm{p}<0.001)$.

Further, postoperative pain scores were reduced up to 12 hours after surgery. ${ }^{89}$ Outcomes were similar in another retrospective study. ${ }^{90}$ Side effects are rare but include infection, pleural injury, vascular injury, pneumothorax, and failure. While other prospective trials are currently underway, further research is warranted to continue outlining the benefits and limitations of ESP blocks. Other local blocks include continuous wound infiltration, targeted nerve root blocks, and local analgesic administered intraoperatively to the skin and subcutaneous tissues.

\section{PRESCRIBING LIMITATIONS}

In the past two decades, opioid use and prescribing have become the focus of state and national legislation. The number of opioid overdose deaths has quadrupled since 1999 and included over 500,000 people. ${ }^{91}$ Legislation targeting prescribing limitations has been enacted in over 35 states since 2016 to regulate the daily and total MMEs of narcotic medications that providers can prescribe. ${ }^{92}$ These laws are of particular interest in spine surgery, as patients facing chronic neck and back pain who undergo surgery are among the heaviest opioid users both preoperatively and postoperatively. ${ }^{93}$ Questions have subsequently been raised about the effectiveness of these laws and the potential for suboptimal pain control that could result in discomfort and complications such as decreased mobility after surgery.
While the evidence evaluating the efficacy and results of these prescribing limitations after spine surgery is limited, several studies have attempted to elucidate information. For example, in 2019, Reid et al. performed a retrospective review of 211 patients undergoing anterior cervical discectomy and fusion surgeries and compared patients who had surgery before the prescribing limitation to those who had surgery after. ${ }^{94}$ Interestingly, patients who had surgery post-law received fewer postoperative MMEs and fewer 30-day postoperative MMEs with no subsequent increase in ED visits or readmissions. Although not specifically investigated, this suggests there was no significant reduction in pain control.

Another retrospective review investigated this question in lumbar spine surgery. It again demonstrated a reduction in postoperative MMEs and 30-day postoperative MMEs with no increase in ED visits or readmissions after prescribing limitation laws. ${ }^{95}$ A recent study comparing nearly 25,000 patients undergoing spine surgery in Michigan also found a $9 \%$ decrease $(p<0.001)$ in preoperative opioid use after prescribing laws were enacted. ${ }^{96}$ Finally, Lovecchio et al. investigated a cohort of patients undergoing lumbar surgery before and after an educational conference, and institutional guidelines on postoperative opioid prescribing were instituted. ${ }^{97}$ These interventions significantly reduced the MMEs prescribed at discharge. While current work is underway to illustrate further the effects of prescribing limitation laws on patients undergoing spine surgery specifically, further prospective research that simultaneously investigates patient comfort, pain scores, and recovery after surgery is warranted.

\section{CONCLUSION}

As spinal conditions and procedures tend to be quite invasive and painful, physicians are tasked with a challenging and delicate balancing act between providing patients adequate pain control and curtailing excessive opioid use. Over the past few decades, the repercussions of excessive opioid use have been detrimental for health systems, communities, and individual patients. Furthermore, with an aging population, spine treatments will almost certainly continue to increase, which places a large responsibility on medical providers to deliver the best pain control possible to their patients without worsening the existing opioid epidemic. While there is undoubtedly room for improvement, the issue has continued to get well-deserved attention resulting in many efforts that have decreased not only opioid consumption but also improved certain patient outcomes. Therefore, further prospective research is warranted to continue implementing best practices by clinicians to continue improving the quality and safety of pain control methods following spine surgery.

\section{DISCLOSURES}

No relevant disclosures. 
FUNDING

Submitted: June 11, 2021 EST, Accepted: June 18, 2021 EST

No funding. 


\section{REFERENCES}

1. Sabatino MJ, Kunkel ST, Ramkumar DB, Keeney BJ, Jevsevar DS. Excess Opioid Medication and Variation in Prescribing Patterns Following Common Orthopaedic Procedures. J Bone Joint Surg Am. 2018;100(3):180-188. doi:10.2106/JBJS.17.00672

2. Kovacs RJ, Gilbert JH, Oetgen WJ. Call to Action: Opioid Crisis Impacting More Than Just Patients. J Am Coll Cardiol. 2020;75(3):341-343. doi:10.1016/j.jac c.2019.12.010

3. Rollston R. Our Medical and Social Responsibility in Addressing the Opioid Epidemic. Med Care. 2020;58(7):582-585. doi:10.1097/MLR.000000000000 $\underline{1349}$

4. Brunette MF, Oslin DW, Dixon LB, et al. The Opioid Epidemic and Psychiatry: The Time for Action Is Now. Psychiatr Serv. 2019;70(12):1168-1171. doi:10.1176/ap pi.ps.201800582

5. Mendoza-Elias N, Dunbar M, Ghogawala Z, Whitmore RG. Opioid Use, Risk Factors, and Outcome in Lumbar Fusion Surgery. World Neurosurg. 2020;135:e580-e587. doi:10.1016/j.wneu.2019.12.073

6. Oliver JE, Carlson C. Misperceptions about the “Opioid Epidemic:” Exploring the Facts. Pain Manag Nurs. 2020;21(1):100-109. doi:10.1016/i.pmn.2019.0 $\underline{5.004}$

7. Jain N, Brock JL, Phillips FM, Weaver T, Khan SN. Chronic preoperative opioid use is a risk factor for increased complications, resource use, and costs after cervical fusion. Spine J. 2018;18(11):1989-1998. doi:1 $\underline{0.1016 / j . s p i n e e .2018 .03 .015}$

8. Grace TR, Choo KJ, Patterson JT, Khanna K, Feeley BT, Zhang AL. A Review of Inpatient Opioid Consumption and Discharge Prescription Patterns After Orthopaedic Procedures. J Am Acad Orthop Surg. 2020;28(7):279-286. doi:10.5435/JAAOS-D-19-00279

9. Orfield NJ, Gaddis A, Russell KB, Hartman DW, Apel PJ, Mierisch C. New Long-Term Opioid PrescriptionFilling Behavior Arising in the 15 Months After Orthopaedic Surgery. J Bone Joint Surg Am. 2020;102(4):332-339. doi:10.2106/JBJS.19.00241

10. Reyes AA, Canseco JA, Mangan JJ, et al. Risk Factors for Prolonged Opioid Use and Effects of Opioid Tolerance on Clinical Outcomes After Anterior Cervical Discectomy and Fusion Surgery. Spine (Phila Pa 1976). 2020;45(14):968-975. doi:10.1097/BRS.0000 $\underline{000000003511}$
11. Adogwa O, Davison MA, Vuong VD, et al. Regional Variation in Opioid Use After Lumbar Spine Surgery. World Neurosurg. 2019;121:e691-e699. doi:10.1016/ j.wneu.2018.09.192

12. Lall MP, Restrepo E. Predictors of Weeks to Opioid Cessation after Lumbar Fusion: A Prospective Cohort Study. Pain Manag Nurs. 2018;19(5):525-534. doi:10.1 016/j.pmn.2018.04.007

13. Sharma M, Ugiliweneza B, Sirdeshpande P, Wang D, Boakye M. Opioid Dependence and Health Care Utilization After Decompression and Fusion in Patients With Adult Degenerative Scoliosis. Spine (Phila Pa 1976). 2019;44(4):280-290. doi:10.1097/BR $\underline{\mathrm{S} .0000000000002794}$

14. Adogwa O, Parker SL, Bydon A, Cheng J, Mcgirt MJ. Comparative Effectiveness of Minimally Invasive Versus Open Transforaminal Lumbar Interbody Fusion 2-Year Assessment of Narcotic Use, Return to Work, Disability, and Quality of Life. 2011;24. www.sp inal disorders.com\%7C479

15. Cheng JS, Park P, Le H, Reisner L, Chou D, Mummaneni PV. Short-term and long-term outcomes of minimally invasive and open transforaminal lumbar interbody fusions: Is there a difference? Neurosurg Focus. 2013;35(2). doi:10.3171/2013.5.FOC US1377

16. Lee MJ, Mok J, Patel P. Transforaminal Lumbar Interbody Fusion: Traditional Open Versus Minimally Invasive Techniques. J Am Acad Orthop Surg. 2018;26(4):124-131. doi:10.5435/JAAOS-D-15-00756

17. Schoenfeld AJ, Nwosu K, Jiang W, et al. Risk Factors for Prolonged Opioid Use Following Spine Surgery, and the Association with Surgical Intensity, among Opioid-Naive Patients. J Bone Jt Surg - Am Vol. 2017;99(15):1247-1252. doi:10.2106/JBJS.16.01075

18. Klueh MP, Hu HM, Howard RA, et al. Transitions of Care for Postoperative Opioid Prescribing in Previously Opioid-Naïve Patients in the USA: a Retrospective Review. J Gen Intern Med. Published online June 11, 2018:1-7. doi:10.1007/s11606-018-44 $\underline{63-1}$

19. Warner NS, Finnie D, Warner DO, et al. The System Is Broken: A Qualitative Assessment of Opioid Prescribing Practices After Spine Surgery. Mayo Clin Proc. 2020;95(9):1906-1915. doi:10.1016/j.mayocp.20 20.02.027 
20. Kalakoti P, Volkmar AJ, Bedard NA, Eisenberg JM, Hendrickson NR, Pugely AJ. Preoperative Chronic Opioid Therapy Negatively Impacts Long-term Outcomes Following Cervical Fusion Surgery. Spine (Phila Pa 1976). 2019;44(18):1279-1286. doi:10.1097/ BRS.0000000000003064

21. Jain N, Phillips FM, Weaver T, Khan SN. Preoperative chronic opioid therapy: A risk factor for complications, readmission, continued opioid use and increased costs after one- and two-level posterior lumbar fusion. Spine (Phila Pa 1976).

2018;43(19):1331-1338. doi:10.1097/BRS.0000000000 $\underline{002609}$

22. Zakaria HM, Mansour TR, Telemi E, et al. The Association of Preoperative Opioid Usage With Patient-Reported Outcomes, Adverse Events, and Return to Work After Lumbar Fusion: Analysis From the Michigan Spine Surgery Improvement Collaborative (MSSIC). Neurosurgery. 2020;87(1):142-149. doi:10.1093/neuros/nyz423

23. Anderson JT, Haas AR, Percy R, Woods ST, Ahn UM, Ahn NU. Chronic opioid therapy after lumbar fusion surgery for degenerative disc disease in a workers' compensation setting. Spine (Phila Pa 1976). 2015;40(22):1775-1784. doi:10.1097/BRS.0000000000 $\underline{001054}$

24. Tank A, Hobbs J, Ramos E, Rubin DS. Opioid dependence and prolonged length of stay in lumbar fusion: A retrospective study utilizing the national inpatient sample 2003-2014. Spine (Phila Pa 1976). 2018;43(24):1739-1745. doi:10.1097/BRS.0000000000 $\underline{002714}$

25. Kha ST, Scheman J, Davin S, Benzel EC. The Impact of Preoperative Chronic Opioid Therapy in Patients Undergoing Decompression Laminectomy of the Lumbar Spine. Spine (Phila Pa 1976). 2020;45(7):438-443. doi:10.1097/BRS.0000000000003 $\underline{297}$

26. Cook DJ, Kaskovich S, Pirkle S, et al. Benchmarks of Duration and Magnitude of Opioid Consumption after Common Spinal Procedures: A Database Analysis of 47,823 Patients. Spine (Phila Pa 1976). 2019;44(23):1668-1675. doi:10.1097/BRS.0000000000 $\underline{003141}$

27. Warner NS, Habermann EB, Hooten WM, et al. Association Between Spine Surgery and Availability of Opioid Medication. JAMA Netw Open.

2020;3(6):e208974. doi:10.1001/jamanetworkopen.20 $\underline{20.8974}$

28. Lo YT, Lim-Watson M, Seo Y, et al. Long-Term Opioid Prescriptions After Spine Surgery: A MetaAnalysis of Prevalence and Risk Factors. World Neurosurg. 2020;141:e894-e920. doi:10.1016/j.wneu.2 $\underline{020.06 .081}$
29. Harris AB, Marrache M, Jami M, et al. Chronic opioid use following anterior cervical discectomy and fusion surgery for degenerative cervical pathology. Spine J. 2020;20(1):78-86. doi:10.1016/j.spinee.2019.0 9.011

30. Pugely AJ, Bedard NA, Kalakoti P, et al. Opioid use following cervical spine surgery: trends and factors associated with long-term use. Spine J. 2018;18(11):1974-1981. doi:10.1016/i.spinee.2018.0 $\underline{3.018}$

31. Karhade AV, Ogink PT, Thio QCBS, et al. Development of machine learning algorithms for prediction of prolonged opioid prescription after surgery for lumbar disc herniation. Spine $J$. 2019;19(11):1764-1771. doi:10.1016/i.spinee.2019.0 $\underline{6.002}$

32. Martini ML, Nistal DA, Deutsch BC, Caridi JM. Characterizing the risk and outcome profiles of lumbar fusion procedures in patients with opioid use disorders: a step toward improving enhanced recovery protocols for a unique patient population. Neurosurg Focus. 2019;46(4). doi:10.3171/2019.1.FOCUS1944

33. Dunn LK, Yerra S, Fang S, et al. Incidence and Risk Factors for Chronic Postoperative Opioid Use After Major Spine Surgery: A Cross-Sectional Study With Longitudinal Outcome. Anesth Analg. 2018;127(1):247-254. doi:10.1213/ANE.000000000000 $\underline{3338}$

34. Sharma M, Ugiliweneza B, Aljuboori Z, Nuno MA, Drazin D, Boakye M. Factors predicting opioid dependence in patients undergoing surgery for degenerative spondylolisthesis: analysis from the MarketScan databases. J Neurosurg Spine. 2018;29(3):271-278. doi:10.3171/2018.1.SPINE171258

35. Adogwa O, Davison MA, Vuong V, et al. Sex Differences in Opioid Use in Patients With Symptomatic Lumbar Stenosis or Spondylolisthesis Undergoing Lumbar Decompression and Fusion. Spine (Phila Pa 1976). 2019;44(13):E800-E807. doi:10.1097/ BRS.0000000000002965

36. Boslett AJ, Denham A, Hill EL, Adams MCB. Unclassified drug overdose deaths in the opioid crisis: emerging patterns of inequity. J Am Med Inform Assoc. 2019;26(8-9):767-777. doi:10.1093/jamia/ocz050

37. Rosenthal BD, Suleiman LI, Kannan A, Edelstein AI, Hsu WK, Patel AA. Risk Factors for Prolonged Postoperative Opioid Use After Spine Surgery: A Review of Dispensation Trends From a State-run Prescription Monitoring Program. J Am Acad Orthop Surg. 2019;27(1):32-38. doi:10.5435/JAAOS-D-17-003 $\underline{04}$ 
38. Kalakoti P, Hendrickson NR, Bedard NA, Pugely AJ. Opioid Utilization Following Lumbar Arthrodesis: Trends and Factors Associated With Long-term Use. Spine (Phila Pa 1976). 2018;43(17):1208-1216. doi:1 0.1097/BRS.0000000000002734

39. Ring D, Sabharwal S. Opioids and Orthopaedics in North America: Addressing a Growing Concern. J Bone Joint Surg Am. 2017;99(22):1881-1882. doi:10.21 $\underline{06 / \mathrm{IBJS} .17 .00880}$

40. Christian ZK, Youssef CA, Aoun SG, et al. Smoking has a dose-dependent effect on the incidence of preoperative opioid consumption in female geriatric patients with spine disease. J Clin Neurosci. 2020;81:173-177. doi:10.1016/j.jocn.2020.09.066

41. Sun EC, Darnall BD, Baker LC, Mackey S. Incidence of and Risk Factors for Chronic Opioid Use Among Opioid-Naive Patients in the Postoperative Period. JAMA Intern Med. 2016;176(9):1286-1293. do i:10.1001/jamainternmed.2016.3298

42. Hills JM, Carlile CR, Archer KR, et al. Duration and Dosage of Opioids After Spine Surgery: Implications on Outcomes at 1 Year. Spine (Phila Pa 1976). 2020;45(15):1081-1088. doi:10.1097/BRS.0000 $\underline{000000003446}$

43. Kehlet H. Multimodal approach to control postoperative pathophysiology and rehabilitation. $\mathrm{Br}$ J Anaesth. 1997;78(5):606-617. doi:10.1093/bja/78.5.6 $\underline{06}$

44. Song W, Wang K, Zhang R jin, Dai Q xin, Zou S bing. The enhanced recovery after surgery (ERAS) program in liver surgery: a meta-analysis of randomized controlled trials. SpringerPlus. 2016;5(1):1-10. doi:10.1186/s40064-016-1793-5

45. Eskicioglu C, Forbes SS, Aarts MA, Okrainec A, McLeod RS. Enhanced recovery after surgery (ERAS) programs for patients having colorectal surgery: A meta-analysis of randomized trials. J Gastrointest Surg. 2009;13(12):2321-2329. doi:10.1007/s11605-00 9-0927-2

46. Ljungqvist O, Scott M, Fearon KC. Enhanced recovery after surgery a review. JAMA Surg. 2017;152(3):292-298. doi:10.1001/jamasurg.2016.495 2

47. Smith TWJ, Wang X, Singer MA, Godellas CV, Vance FT. Enhanced recovery after surgery: A clinical review of implementation across multiple surgical subspecialties. Am J Surg. 2020;219(3):530-534. doi:1 $\underline{0.1016 / \text { j.amjurg.2019.11.009 }}$
48. Lemanu DP, Singh PP, Stowers MDJ, Hill AG. A systematic review to assess cost effectiveness of enhanced recovery after surgery programmes in colorectal surgery. Color Dis. 2014;16(5):338-346. do i:10.1111/codi.12505

49. Miller TE, Thacker JK, White WD, et al. Reduced length of hospital stay in colorectal surgery after implementation of an enhanced recovery protocol. Anesth Analg. 2014;118(5):1052-1061. doi:10.1213/A NE.0000000000000206

50. Jeong O, Ryu SY, Park YK. Postoperative Functional Recovery after Gastrectomy in Patients Undergoing Enhanced Recovery after Surgery. Med (United States). 2016;95(14):1-6. doi:10.1097/MD.000 $\underline{0000000003140}$

51. Rajaee S, Kanim L, Delamarter RB, Bae HW. A Careful Analysis of Trends in Spinal Fusion in the United States from 1998 to 2008. 1998;(642):2011.

52. Archer K, Bird ML, Haug C, et al. Patients' Experience and Expectations of Lumbar Spine Surgery for Degenerative Conditions: A Qualitative Study. Spine J. 2015;15(10):S99-S100. doi:10.1016/j.sp inee.2015.07.046

53. Dietz N, Sharma M, Adams S, et al. Enhanced Recovery After Surgery (ERAS) for Spine Surgery: A Systematic Review. World Neurosurg.

2019;130:415-426. doi:10.1016/j.wneu.2019.06.181

54. Debono B, Wainwright TW, Wang MY, et al. Consensus statement for perioperative care in lumbar spinal fusion: Enhanced Recovery After Surgery (ERAS ${ }^{\circledR}$ ) Society recommendations. Spine J. 2021;000. doi:10.1016/j.spinee.2021.01.001

55. Krebs EE, Lurie JD, Fanciullo G, et al. Predictors of Long-Term Opioid Use Among Patients With Painful Lumbar Spine Conditions. J Pain. 2010;11(1):44-52. $\underline{\mathrm{d}}$ oi:10.1016/j.jpain.2009.05.007

56. Liu B, Liu R, Wang L. A meta-analysis of the preoperative use of gabapentinoids for the treatment of acute postoperative pain following spinal surgery. Med (United States). 2017;96(37):1-11. doi:10.1097/M D.0000000000008031

57. Han C, Kuang MJ, Ma JX, Ma XL. The efficacy of preoperative gabapentin in spinal surgery: A Metaanalysis of randomized controlled trials. Pain Physician. 2017;20(7):649-661.

58. Araimo Morselli FSM, Zuccarini F, Caporlingua F, et al. Intrathecal Versus Intravenous Morphine in Minimally Invasive Posterior Lumbar Fusion: A Blinded Randomized Comparative Prospective Study. Spine (Phila Pa 1976). 2017;42(5):281-284. doi:10.109 7/BRS.0000000000001733 
59. Kang H, Jung HJ, Lee JS, Yang JJ, Shin HY, Song KS. Early postoperative analgesic effects of a single epidural injection of ropivacaine administered preoperatively in posterior lumbar interbody spinal arthrodesis a pilot randomized controlled trial. J Bone Jt Surg - Ser A. 2013;95(5):393-399. doi:10.2106/JBI S.K.01729

60. Park SY, An HS, Lee SH, Suh SW, Kim JL, Yoon SJ. A prospective randomized comparative study of postoperative pain control using an epidural catheter in patients undergoing posterior lumbar interbody fusion. Eur Spine J. 2016;25(5):1601-1607. doi:10.100 7/s00586-016-4385-8

61. Jirarattanaphochai K, Jung S, Thienthong S, Krisanaprakornkit W, Sumananont C. Peridural methylprednisolone and wound infiltration with bupivacaine for postoperative pain control after posterior lumbar spine surgery: A randomized double-blinded placebo-controlled trial. Spine (Phila Pa 1976). 2007;32(6):609-616. doi:10.1097/01.brs.000 0257541.91728.a1

62. Soffin EM, Wetmore DS, Barber LA, et al. An enhanced recovery after surgery pathway: association with rapid discharge and minimal complications after anterior cervical spine surgery. Neurosurg Focus. 2019;46(4):E9. doi:10.3171/2019.1.FOCUS18643

63. Grundmann U, Wörnle C, Biedler A, Kreuer S, Wrobel M, Wilhelm W. The efficacy of the non-opioid analgesics parecoxib, paracetamol and metamizol for postoperative pain relief after lumbar microdiscectomy. Anesth Analg. 2006;103(1):217-222. doi:10.1213/01.ane.0000221438.08990.06

64. Feng C, Zhang Y, Chong F, et al. Establishment and Implementation of an Enhanced Recovery After Surgery (ERAS) Pathway Tailored for Minimally Invasive Transforaminal Lumbar Interbody Fusion Surgery. World Neurosurg. 2019;129:e317-e323. doi:1 $\underline{0.1016 / \text { i.wneu.2019.05.139 }}$

65. Benyamin R, Trescot AM, Datta S, et al. Opioid complications and side effects. Pain Physician. 2008;11(SPEC. ISS. 2):105-120.

66. Jamison RN, Dorado K, Mei A, Edwards RR, Martel MO. Influence of opioid-related side effects on disability, mood, and opioid misuse risk among patients with chronic pain in primary care. Pain Reports. 2017;2(2):1-7. doi:10.1097/PR9.00000000000 $\underline{00589}$

67. Dunn KM, Saunders KW, Rutter CM, et al. Opioid prescriptions for chronic pain and overdose: A cohort study. Ann Intern Med. 2010;152(2):85-92. doi:10.732 6/0003-4819-152-2-201001190-00006
68. Ghanem CI, Pérez MJ, Manautou JE, Mottino AD. Acetaminophen from liver to brain: New insights into drug pharmacological action and toxicity. Pharmacol Res. 2016;109:119-131. doi:10.1016/j.phrs.2016.02.02 $\underline{0}$

69. Davies NM. Clinical pharmacokinetics of ibuprofen. The first 30 years. Clin Pharmacokinet. 1998;34(2):101-154. doi:10.2165/00003088-19983402 $\underline{0-00002}$

70. Stahl SM, Porreca F, Taylor CP, Cheung R, Thorpe AJ, Clair A. The diverse therapeutic actions of pregabalin: Is a single mechanism responsible for several pharmacological activities? Trends Pharmacol Sci. 2013;34(6):332-339. doi:10.1016/j.tips.2013.04.00 1

71. Takasusuki T, Yaksh TL. The effects of intrathecal and systemic gabapentin on spinal substance $P$ release. Anesth Analg. 2011;112(4):971-976. doi:10.12 13/ANE.0b013e31820f2a16

72. Derry S, Moore RA. Topical lidocaine for neuropathic pain in adults. Cochrane Database Syst Rev. 2014;2014(2). doi:10.1002/14651858.CD010958

73. Walker CT, Gullotti DM, Prendergast V, et al. Implementation of a Standardized Multimodal Postoperative Analgesia Protocol Improves Pain Control, Reduces Opioid Consumption, and Shortens Length of Hospital Stay After Posterior Lumbar Spinal Fusion. Neurosurgery. 2020;87(1):130-136. doi:10.109 3/neuros/nyz312

74. Garcia RM, Cassinelli EH, Messerschmitt PJ, Furey CG, Bohlman HH. A multimodal approach for postoperative pain management after lumbar decompression surgery: A prospective, randomized study. J Spinal Disord Tech. 2013;26(6):291-297. doi:1 $\underline{0.1097 / B S D .0 b 013 e 318246 b 0 a 6}$

75. Mathiesen O, Dahl B, Thomsen BA, et al. A comprehensive multimodal pain treatment reduces opioid consumption after multilevel spine surgery. Eur Spine J. 2013;22(9):2089-2096. doi:10.1007/s0058 6-013-2826-1

76. Rajpal S, Gordon DB, Pellino TA, et al. Comparison of perioperative oral multimodal analgesia versus IV PCA for spine surgery. J Spinal Disord Tech. 2010;23(2):139-145. doi:10.1097/BSD.0b 013e3181cf07ee

77. Breebaart MB, Van Aken D, De Fre O, et al. A prospective randomized double-blind trial of the efficacy of a bilateral lumbar erector spinae block on the $24 \mathrm{~h}$ morphine consumption after posterior lumbar inter-body fusion surgery. Trials. 2019;20(1):441. doi:10.1186/s13063-019-3541-y 
78. Sucato DJ, Duey-Holtz A, Elerson E, Safavi F. Postoperative analgesia following surgical correction for adolescent idiopathic scoliosis: A comparison of continuous epidural analgesia and patient-controlled analgesia. Spine (Phila Pa 1976). 2005;30(2):211-217. doi:10.1097/01.brs.0000150832.53604.64

79. Alican MFB, Ver MR, Ramos MRD, Mamaril LJC. Postoperative Single-shot Epidural Fentanyl and Bupivacaine for Postoperative Analgesia After Lumbar Decompression: A Prospective, Double-blind Randomized Study. Spine (Phila Pa 1976). 2020;45(15):1017-1023. doi:10.1097/BRS.0000000000 $\underline{003449}$

80. Sharma S, Balireddy RK, Vorenkamp KE, Durieux ME. Beyond opioid patient-controlled analgesia: A systematic review of analgesia after major spine surgery. Reg Anesth Pain Med. 2012;37(1):79-98. doi:1 0.1097/AAP.0b013e3182340869

81. Lotfinia I, Khallaghi E, Meshkini A, Shakeri M, Shima M, A.S. Interaoperative use of epidural methylprednisolone or bupivacaine for postsurgical lumbar discectomy pain relief: A randomized, placebo-controlled trial. Ann Saudi Med. 2007;27(4).

82. Jellish WS, Abodeely A, Fluder EM, Shea J. The effect of spinal bupivacaine in combination with either epidural clonidine and/or 0.5\% Bupivacaine administered at the incision site on postoperative outcome in patients undergoing lumbar laminectomy. Anesth Analg. 2003;96(3):874-880. doi:10.1213/01.AN E.0000049682.48703.44

83. Bourke DL, Spatz E, Motara R, Ordia JI, Reed J, Hlavacek JM. Epidural opioids during laminectomy surgery for postoperative pain. J Clin Anesth. 1992;4(4):277-281. doi:10.1016/0952-8180(92)9012 $\underline{8-\mathrm{N}}$

84. Dhaliwal P, Yavin D, Whittaker T, et al. Intrathecal Morphine Following Lumbar Fusion: A Randomized, Placebo-Controlled Trial. Neurosurgery.

2019;85(2):189-198. doi:10.1093/neuros/nyy384

85. Adamczyk W, Tafil-Klawe M, Chęsy G, Klawe JJ, Szeliga-Wczysła M, Złomańczuk P. Effects of training on the ventilatory response to hypoxia. J Physiol Pharmacol. 2006;57(SUPPL. 4):7-14.

86. Chan JHH, Heilpern GNA, Packham I, Trehan RK, Marsh GDJ, Knibb AA. A prospective randomized double-blind trial of the use of intrathecal fentanyl in patients undergoing lumbar spinal surgery. Spine (Phila Pa 1976). 2006;31(22):2529-2533. doi:10.1097/ 01.brs.0000241135.79983.52
87. Khan ZH, Hamidi S, Miri M, Majedi H, Nourijelyani K. Post-operative pain relief following intrathecal injection of acetylcholine esterase inhibitor during lumbar disc surgery: A prospective double blind randomized study. Spine (Phila Pa 1976). 2008;33(6):669-675. doi:10.1111/j.1365-2710.2008.00 968.x

88. Forero M, Adhikary SD, Lopez H, Tsui C, Chin KJ. The erector spinae plane block a novel analgesic technique in thoracic neuropathic pain. Reg Anesth Pain Med. 2016;41(5):621-627. doi:10.1097/AAP.0000 $\underline{000000000451}$

89. Abu Elyazed MM, Mostafa SF, Abdelghany MS, Eid GM. Ultrasound-Guided Erector Spinae Plane Block in Patients Undergoing Open Epigastric Hernia Repair: A Prospective Randomized Controlled Study. Anesth Analg. 2019;129(1):235-240. doi:10.1213/ANE.000000 $\underline{0000004071}$

90. Ueshima $\mathrm{H}$, Inagaki M, Toyone T, Otake $\mathrm{H}$. Efficacy of the erector spinae plane block for lumbar spinal surgery: A retrospective study. Asian Spine J. 2019;13(2):254-257. doi:10.31616/ASJ.2018.0114

91. Centers for Disease Control and Prevention. Opioid Overdose: Understanding the Epidemic.; 2021.

92. Reid DBC, Patel SA, Shah KN, et al. Opioidlimiting legislation associated with decreased 30-day opioid utilization following anterior cervical decompression and fusion. Spine J. 2020;20(1):69-77. doi:10.1016/j.spinee.2019.08.014

93. Martin BI, Deyo RA, Mirza SK, et al. Expenditures and health status among adults with back and neck problems. JAMA - J Am Med Assoc. 2008;299(6):656-664. doi:10.1001/jama.299.6.656

94. Reid DBC, Patel SA, Shah KN, et al. Opioidlimiting legislation associated with decreased 30-day opioid utilization following anterior cervical decompression and fusion. Spine J. 2020;20(1):69-77. doi:10.1016/i.spinee.2019.08.014

95. Reid DBC, Shah KN, Ruddell JH, et al. Effect of narcotic prescription limiting legislation on opioid utilization following lumbar spine surgery. Spine J. 2019;19(4):717-725. doi:10.1016/j.spinee.2018.09.007

96. Park P, Chang V, Yeh HH, et al. Impact of Michigan's new opioid prescribing laws on spine surgery patients: Analysis of the Michigan spine surgery improvement collaborative. J Neurosurg Spine. 2021;34(3):531-536. doi:10.3171/2020.7.SPINE20729 
97. Lovecchio F, Stepan JG, Premkumar A, et al. An institutional intervention to modify opioid

prescribing practices after lumbar spine surgery. $J$

Neurosurg Spine. 2019;30(4):483-490. doi:10.3171/201

8.8.SPINE18386 